\title{
Energy absorption evaluation of reinforced concrete beams under various loading rates based on particle swarm optimization technique
}

\begin{abstract}
This study proposes an energy absorption model for predicting the effect of loading rates, concrete compressive strength, shear span-to-depth ratio, and longitudinal and transverse reinforcement ratio of reinforced concrete (RC) beams using the particle swarm optimization (PSO) technique. This technique avoids the exhaustive traditional trial-and-error procedure for obtaining the coefficient of the proposed model. Fifty-six RC slender and deep beams are collected from the literature and used to build the proposed model. Three performance measures, namely, mean absolute error, mean absolute percentage error and root mean square error, are investigated in the proposed model to increase its accuracy. The design procedure and accuracy of the proposed model are illustrated and analysed via simulation tests in a MATLAB/Simulink environment. The results indicate the minimal effect of swarm size on the convergence of the PSO algorithm, as well as the ability of PSO to search for an optimum set of coefficients from within the solution space.
\end{abstract}

Keyword: Energy absorption; Concrete beam; Particle swarm optimization (PSO) 